\title{
La Riqueza Cultural, Ecológica para fomentar Actividades Turísticas ${ }^{1}$
}

\section{The Cultural Richness, Ecological to Promote Tourist Activities}

DOI: http://dx.doi.org/10.17981/cultedusoc.9.2.2018.05

Fecha de recepción: 20/06/2018. Fecha de aceptación: 14/09/2018

Victoria Dávila-Pertuz ${ }^{2}$

Yadira Ibáñez; Rocío Chacón; Mariana Mercado; Humberto Aragón; Edith González; Nays Hernández ${ }^{3}$

Para citar este artículo

Dávila-Pertuz, V., Ibáñez, Y., Chacón, R., Mercado, M., Aragón, H., González, E., y Hernández, N. (2018). La Riqueza Cultural, Ecológica para fomentar Actividades Turísticas. Cultura. Educación y Sociedad 9(2), 56-66. DOI: http://dx.doi.org/10.17981/cultedusoc.9.2.2018.05

\section{Resumen}

La investigación tiene como propósito determinar el grado de conocimiento que tienen de su entorno, de los sitios que nos permiten develar su historia, los que nos permiten recrearnos, etc. Con esta información, los investigadores inician un rastreo bibliográfico para ahondar del tema del Ecoturismo, también de actividades lúdicas y recreativas que puedan resultar atractivas para los visitantes. La investigación es de corte Cualitativo, descriptiva. La técnica de recolección fue la entrevista y la observación, Los participantes en la aplicación han sido 5 docentes y 20 estudiantes de los grados $10^{\circ}$ y $11^{\circ}$, de la Institución Educativa Roque de los Ríos Valle. Como conclusiones se puede inferir que son los docentes los que deben indagar sobre todos los sitios de interés con que cuenta el municipio, apropiarse de esta información y compartirla con los estudiantes para motivarlos a expandir su horizonte fijándose metas de desarrollo personal y comunitario.

Palabras clave: Ecoturismo; turismo rural comunitario; tradición, patrimonio, cultura, aprendizajes

\begin{abstract}
The purpose of the research is to determine the degree of knowledge they have about their environment, the places that allow us to reveal their history, the ones that allow us to recreate ourselves, etc. With this information, the researchers begin a bibliographic search to delve into the topic of Ecotourism, as well as recreational and recreational activities that may be attractive to visitors. The investigation is qualitative, descriptive. The collection technique was the interview and the observation. The participants in the application were 5 teachers and 20 students from the 10th and 11th grades, from the Educational Institution Roque de los Ríos Valle. As conclusions, it can be inferred that it is the teachers who should inquire about all the sites of interest that the municipality has, appropriate this information and share it with the students to motivate them to expand their horizon, setting personal and community development goals.
\end{abstract}

Keywords: Ecotourism; rural community tourism; tradition, heritage, culture, learning

\footnotetext{
${ }^{1}$ Este artículo ha sido derivado del Programa de Fortalecimiento de la Cultura Ciudadana y Democrática CT+I a través de la IEP apoyada en TIC en el Departamento de Magdalena: CICLON.

${ }^{2}$ Líder del grupo de investigación "Maestros exploradores del Reten". Correspondencia: Email: mavila04@hotmail.com

${ }^{3}$ Docentes pertenecientes al grupo de investigación "Maestros exploradores del Reten" de la IED Roque de los Ríos Valle. Ciclón Magdalena, estrategia $\mathrm{N}^{\circ} 2$. Universidad de la Costa CUC.
}

- The author; licensee Universidad de la Costa - CUC. 


\section{Introducción}

Actualmente, el turismo representa una demandante apertura en el mercado, por su posibilidad manifiesta para la generación de empleo, tomando como esencial la satisfacción de los clientes, haciendo referencia a los resultados de un servicio prestado en cualquier lugar del mundo. (Reyes, Marín y Gutierrez 2006).

El Turismo Rural Comunitario, para las comunidades rurales que están ubicadas cerca de atractivos lugares turísticos, puede representa una oportunidad de generación de ingresos y de desarrollo, teniendo en cuenta que aún no se benefician económicamente de los que visitan estos lugares; cada vez está siendo más utilizado por Agencias y ONG como estrategia de desarrollo, así como por el sector público local en cuanto a la gestión del territorio, los recursos y el patrimonio.

El Reten es un municipio ubicado en la zona Norte del Departamento del Magdalena, su posición dentro de la geografía nacional es privilegiada, ya que está en medio de dos ríos caudalosos como son el rio Aracataca y el rio Fundación, además, cuenta con otras corrientes menores como el río Ají y otros, lo cual hace de este territorio un lugar fértil, donde se desarrolla una flora abundante y variada que genera hermosos paisajes, conformada por fuertes y robustos árboles maderables, arbustos grandes y pequeños, hasta llegar a rastreras con diversidad de flores que visten la estancia con vistosos colores y que, además, constituyen el hábitat ideal para muchas especies animales, o sea que también tiene una rica fauna. Se puede mencionar entre otras la gran variedad de aves y animales de caza menor, aves de corral lo mismo que ganado vacuno, equino, porcino etc.
Es un municipio del departamento del Magdalena, relativamente joven e inexplorado en lo que a turismo se refiere. Toda esta riqueza que se menciona, está en grave peligro de extinción debido al desconocimiento que tienen gran parte de los pobladores acerca del aprovechamiento de ella; una práctica muy generalizada, por ejemplo, es la caza de pájaros; niños y jóvenes se dedican a capturar las diferentes especies de pájaros cantores para tenerlos cautivos y venderlos a coleccionistas de estos animalitos. Por otro lado, la flora es afectada por la tala irracional de los maderables y la quema, una práctica tradicional para la adecuación de terrenos para la siembra.

Si no se inician acciones efectivas de pedagogía para la protección de la fauna y la flora, no podremos dejar a las generaciones futuras, este legado tan importante. Se hace necesario emprender, desde las instituciones educativas, programas de información y formación para que no se destruya el entorno sino por el contrario protegerlo, mejorarlo, adecuarlo y optimizarlo para que se convierta en un atractivo para moradores y visitantes.

\section{El Patrimonio, las Tradiciones Culturales y la identidad}

Como lo expone Marcos Arévalo, J. (s.f) "Tradición, Patrimonio e Identidad son conceptos complejos, ambiguos y polisémicos; porque son construcciones sociales cuyos significados cambian dependiendo de la época, el tiempo histórico y según quienes los empleen y para qué fines los utilicen." "Tres vocablos con múltiples acepciones que aluden, cada uno de ellos, a diversas y variadas realidades dependiendo del valor que les confieren historiadores, folkloristas, sociólogos, lingüistas, antropólogos, etc."; "La cultura, los estilos de vida materiales, sociales e 
ideacionales de los pueblos, las sociedades y los grupos humanos, es a la vez universal y particular, múltiple y diversa en la experiencia humana."

Para Esmoris (s.f) "el patrimonio comprende la herencia compartida entre personas, de bienes materiales (inmuebles ó muebles) e inmateriales que son seleccionados por las dimensiones, del sentido de identidad, lo estéticos / artísticos y etnográficos." Si los bienes que se poseen, tienen historia puede ser considerado patrimonial, pues como explica el autor "la Distancia temporal es imprescindible para que haya patrimonio. Se puede sostener que el primer bien patrimonial de una comunidad (mundial, nacional, regional, local), los constituyen sus propios relatos históricos." (p.p, 10). Es la historia, o mejor dicho el paso del Tiempo convertido en narraciones, lo que crea sentido patrimonial, si se describe dentro de una historia (mundial, regional, nacional, local), así como relato y documentación de su historia: "no hay patrimonio sin historia y esa historia debe estar contextualizada."

Además, tiene que tener sus valores basados en su patrimonio cultural para distinguirse por: representar testimonios de épocas y estilos; por excepcionalidad; por poseer atributos singulares.

El patrimonio cultural en un sentido amplio se puede establecer por: "relatos históricos sobre acontecimientos diversos, bienes arqueológicos, arquitectónicos, archivos y acervos, obras de arte, mobiliario, expresiones artísticas, máquinas, artefactos y objetos singulares y/o representativos de actividades, épocas y estilos." (Esmoris M. s.f)

En este sentido, los "bienes etnográficos se distinguen por pertenecer a formas de vida de grupos definidos por una actividad o alguna otra circunstancia o experiencia, que pueda objetivarse en una narración histórica y cuyos componentes materiales e inmateriales primordiales o constitutivos sean pasibles de inventariar", por ejemplo, las costumbres de la colectividad. (Esmoris M. s.f).

En cuanto a tradiciones culturales para la UNESCO, forman parte del patrimonio inmaterial. Las prácticas tradicionales son intangibles y son adquiridas por el individuo mediante la experiencia, de generación en generación, en la propia ejecución, en la actualización de la memoria de procedimientos que no están registrados materialmente. En conclusión el patrimonio inmaterial, no es más que las experiencias que adquiere la persona y aquello que es intangible. "La tradición no se hereda genéticamente; se transmite socialmente y deriva de un proceso de selección cultural. La parte de la cultura seleccionada en el tiempo con una función de uso en el presente sería la tradición. El pasado, decantado, es continuamente reincorporado al presente." (Arévalo, s.f)

"Se trata de bienes y prácticas con
historia, y por esto se lo considera pa-
trimoniales. Son herencia compartida
con significación: artístico-estética,
portadora de sentido de identidad o
valor etnográfico. Es el caso de las
fiestas, las lenguas (los dialectos y
los modismos), las artesanías, la gas-
tronomía, etcétera." (Esmoris M. s.f)

Para (Esmoris s.f): "Además de los procedimientos inmateriales existen objetos, instrumentos y una geografía o entorno material donde se desarrollan y que forman parte del bien patrimonial, conformando un paisaje cultural. También integran las tradiciones culturales, el cultivo del apego a bienes, lugares, vivencias" (p.p 24). "Se debe exponer, que en el origen y en parte también en la ejecución contemporánea, los protagonistas 
principales de las tradiciones fueron, y son, personas con una intuitiva densidad cultural capaz de haber generado y logrado que se reproduzcan a lo largo del tiempo bienes de identidad, originalidad y valor estético artístico."

Siguiendo lo anteriormente planteado, "la identidad consiste en la interiorización por un grupo dado de que posee formas de vida específicas" (p.p 32).

\begin{abstract}
"La identidad, se estructura a partir de la alteridad, en el contraste cultural. Patrimonio e identidad son reflexiones sobre el pasado y la realidad presente. Ahora bien, como construcciones históricas, sociales y culturales, las nociones de patrimonio e identidad se revisan en cada momento histórico. En diferentes períodos temporales la valoración que se hace de uno y otro concepto cambia significativamente." (Marcos Arévalo, J. s.f)
\end{abstract}

Para Marcos Arévalo, J. (s.f) "La identidad, por otra parte, es resultado de un hecho objetivo (el determinante geográfico-espacial, los datos históricos, las específicas condiciones socioeconómicas) y una construcción de naturaleza subjetiva (la dimensión metafísica de los sentimientos y los afectos, la propia experiencia vivencial, la conciencia de pertenencia a un universo local o de otro nivel de integración sociocultural, la tradición, el capital cultural y la específica topografía mental que representan rituales, símbolos y valores)." Así se constituye la identidad.

"Los estudios en turismo son relativamente recientes, si se los compara con la evolución de otras disciplinas de las ciencias sociales, pues solo han pasado 120 años aproximadamente desde que apareció la obra de Stradner en 1884 quien se ocupó por primera vez del estudio de los establecimientos y medios de prestación de servicios." (Campodónico, R. \& Chalar, L. 2010)
Como bien lo ha señalado (Cesar Capanegra 2007, p.2), "los primeros estudios turísticos están signados por la marca del período neoclásico, en el cual se desarrolla la economía como ciencia poniendo especial énfasis en la demanda, el consumo y los consumidores, así como el papel que juega el individuo en la economía." En este punto se llevó a cabo un análisis de la economía y su relación con la oferta y la demanda, teniendo en cuenta que acciones se ejecutaran para que los productores incrementen las ganancias y los consumidores la utilidad.

Es en este marco conceptual que se logran los primero estudios significativos de turismo como es el caso de J. Stradner en 1884 y H.Von Shullen en 1911, que sientan las bases del análisis de la demanda turística y, en 1942, Hunziker - Kraft crean la llamada en su momento "doctrina general del turismo" articulando los elementos objetivos con los subjetivos. Fue en el año 1980 que los estudios universitarios en turismo comienzan a consolidarse en las diferentes instituciones de enseñanza superior del Sur del continente.

Con relación a los periodos que ha afrontado las ciencias sociales (Carrizo, Espina Y Klein, 2004, p.9-18), esta incorporación en los estudios universitarios coincide con la llamada etapa de "crisis de las ciencias sociales" que se llevó a cabo entre 1970 y 1990, la característica más relevante fue que ninguna gran teoría tenía la capacidad de explicar de los nuevos procesos. Esto obedece a la creciente complejidad de las sociedades contemporáneas, donde incrementan y están presentes diversos actores sociales, donde se producen fuertes procesos de multiculturalidad e hibridación que se asocian a la globalización de las relaciones socioeconómicas. Esta crisis es- 
taría marcando los límites de alcance y funcionalidad de la perspectiva de la simplicidad. En este trayecto se han ido consolidando grupos de investigadores y docentes que intentan ir construyendo un corpus de investigación en turismo para conseguir el pleno reconocimiento académico de sus pares ya sea como disciplina o para expresarlo correctamente como campo disciplinario. "Si bien esa parece ser la meta, no es un logro menor en el camino generar sistemáticamente conocimiento científico sobre la actividad turística." (Capanegra, C. 2007).

Según informes del (Ministerio de Comercio, Industria y Turismo, s.f) en Colombia "el Turismo Comunitario representa una alternativa de desarrollo que genera beneficios económicos a las comunidades a su vez que trabaja por la preservación de los recursos naturales y valorización del patrimonio, los derechos económicos, sociales y culturales de sus habitantes."
"El turismo comunitario en Colombia, es desarrollado en zonas rurales y en ocasiones zonas urbanas, posibilita la interacción de las comunidades, por lo general grupos étnicos y familias campesinas, con sus visitantes, permitiéndoles ejercer un papel protagónico en su planificación y gestión, al igual que participan de la distribución de sus beneficios y/o utilidades." (Ministerio de Comercio, Industria y Turismo, s.f)

Esta tendencia del desarrollo turístico según Guerrero (2015) "se caracteriza por la preservación de los recursos naturales y valorización del patrimonio, los derechos económicos, sociales y culturales de sus habitantes."

\section{Ecoturismo}

"El origen del ecoturismo o turismo ecológico como concepto, se remonta a la década de los 80 , cuando la sociedad mundial empezó a hacerse eco de esta forma de turismo como una alternativa viable

\section{Importancia del Turismo Rural Comunitario}

(1945): "El turismo rural nace después de 1945 y se fortalece siempre que hay crisis en el campo: cuando la agricultura peligra, cuando hay crisis económica y en el caso de Colombia, como saneamiento de la violencia. El turismo rural sirve para cambiar el rol de la mujer campesina y las oportunidades de los jóvenes. Siempre que se vincule a la comunidad en todos los procesos de investigación, planeación y por supuesto ejecución, se brinda una oportunidad consensuada para desarrollo económico." (Forum Natura, 2011; Burbano, A. 2011)

Turismo comunitario: "Aprovechando el patrimonio intelectual de la comunidad pueden surgir historias alrededor de los recursos naturales y de la vida cotidiana de las comunidades, storytelling en toda su expresión. Hay que entender el turismo comunitario rural, o mejor aún, turismo en áreas rurales o naturales, como una manifestación cultural y local, lo cual hace a cada destino único y puede ser un factor de mejoramiento de la calidad de vida de los residentes siempre que se retomen los lugares como actores centrales y existan procesos de formación y representaciones sociales." (Burbano, A. 2011; Burgos Doria, R. 2016).

Turismo Natural: "Es necesario explicar a las comunidades que el turismo necesita un marco conceptual, deben hacerse investigaciones y estudios. No todos podemos ser destinos turísticos y no puede ser la única alternativa económica. Si una comunidad agrícola abandona por completo su actividad para dedicarse al turismo pone en riesgo su propia supervivencia. No podemos pensar solamente en el turista, ya que no siempre va a ser el turismo la solución a las necesidades de la comunidad." (Burbano, A. 2011).

Fuente: Elaboración propia (2018). 
de desarrollo sostenible, especialmente como forma de luchar contra el cambio climático" (Vargas Montes, G. 2006).

El ecoturismo puede ser analizada como una estrategia para captar la atención del viajero y exaltar la belleza natural que tiene el país, para promover el sentido de pertenencia por estos lugares, evitar los malos usos, trabajar por la preservación. Todos podemos colaborar en esto, promoviendo el ecoturismo como una alternativa de viaje con la cual podemos respetar y conocer profundamente los límites necesarios que existen para la preservación y cuidado de la naturaleza, "limites que buscan por encima de todo el respeto de nuestro entorno, aprender de los ecosistemas, disfrutar la buena energía y vistas maravillosas que nos ofrece la naturaleza, el equilibrio entre los que conforman estos lugares maravillosos, sagrados como nos enseñan nuestros hermanos indígenas." (Vargas Montes, G. 2006; s.f; Marulanda López, 2012)

Con el ecoturismo se pueden realizar una variedad de actividades las cuales deben estar encaminadas a la preservación y el fomento de los recursos naturales. Teniendo en cuenta las zonas visitadas, serán las actividades que se puedan realizar, sin embargo, podemos destacar claramente las más importantes.

Una actividad que se puede realizar es la observación de ecosistemas, que pueden tomarse como actividades de ocio, la cual puede ser desarrollada con un enfoque analítico, es decir, analizando las diferente funciones que desempeñan los elementos hacen parte de dichos ecosistemas, que pueden ser uno o varios a la vez. La observación es una actividad que propicia la creatividad y para participar en ella no es necesario ser un experto, el objetivo de esta actividad de observación es apreciar la vida animal en el habitad natural.

\section{Metodología}

El tipo de investigación, de acuerdo al enfoque cualitativo del presente proyecto, es etnográfico, puesto que se interesa por el quehacer humano, como este interactúa y se comporta y enfatizar sobre las cuestiones descriptivas e interpretativas de un ámbito sociocultural concreto. Permite el registro del conocimiento cultural, detalla patrones de interacción social, permite el análisis holístico de sociedades, es descriptiva y permite desarrollar y verificar teorías. (Murillo \& Martínez-Garrido, 2010). Es descriptivo, porque el producto final de la investigación es una rica y densa enunciación cualitativa del discurso en la interacción de la comunidad educativa frente al turismo y las tradiciones culturales e históricas del Municipio de El Reten Magdalena.

\section{Escenarios y actores}

Los participantes en la aplicación han sido 5 docentes y 20 estudiantes de los grados $10^{\circ} \mathrm{y} 11^{\circ}$, entre mujeres y hombre, con edades comprendidas de 16 a 50 años, de la Institución Educativa Roque de los Ríos Valle en El Reten Magdalena.

\section{Técnicas e instrumentos de recolección de la información}

Los instrumentos aplicados, para el estudio fue la entrevista estructurada y la observación participante, para ser contrastados con los postulados teóricas establecidos (Jiménez, 2012). 


\section{Procedimiento}

La ejecución de la presente investigación está enmarcada en los siguientes momentos:

Momento (1): Elección del fenómeno a estudiar.

Momento (2): Hacer el sondeo diagnóstico de los objetos de interés.

Momento (3): Definición de las categorías de estudio: Turismo rural comunitario; entorno cultural; entorno ecológico y entorno turístico.

Momento (4): Definición de los instrumentos de recolección de la información.

Momento (5): Aplicación de la entrevista estructurada y los registros de observación. Momento (6): Sistematización de la información a la luz de los datos recolectados y articulación con la teoría.
Momento (7): Generación de resultados.

Momento (8): Socialización de los hallazgos encontrados.

\section{Resultados}

A continuación, se presentará un análisis de la información producto de los resultados obtenidos en función de los objetivos trazados en la presente investigación. Orientado a responder la siguiente pregunta: ¿Cómo a través de la implementación del Turismo Rural Comunitario, la comunidad educativa de la IED Roque de los Ríos Valle, puede conocer, valorar y aprovechar su entorno cultural, ecológico y turístico?

\begin{tabular}{|c|c|c|c|}
\hline $\begin{array}{l}\text { Categoría } \\
\text { de estudio }\end{array}$ & $\begin{array}{l}\text { Pregunta } \\
\text { orientadora }\end{array}$ & $\begin{array}{l}\text { Discurso de } \\
\text { los actores }\end{array}$ & Articulación y sistematización teórica \\
\hline $\begin{array}{l}\text { Entorno } \\
\text { cultural }\end{array}$ & $\begin{array}{l}\text { ¿Cuáles son } \\
\text { las tradiciones } \\
\text { culturales e } \\
\text { históricas del } \\
\text { Municipio de } \\
\text { El Reten? }\end{array}$ & $\begin{array}{l}\text { "las históricas semanas } \\
\text { santas, las fiestas } \\
\text { patronales de San Juan } \\
\text { Bautista, los carnavales y } \\
\text { los encuentros culturales " } \\
\text { las fiestas municipales que } \\
\text { se realizan en el mes de } \\
\text { junio", "la celebración de } \\
\text { la inmaculada concepción” } \\
\text { "las fiestas del barrio" "el } \\
7 \text { de diciembre las fiestas } \\
\text { de San Miguel" "el } 1 \text { de } \\
\text { diciembre el festival del } \\
\text { rio, además de las fiestas } \\
\text { de la hondura, el Bongo y } \\
\text { los olivos corregimientos } \\
\text { del Reten y concursos de } \\
\text { danza” "los paseos" "en el } \\
\text { municipio hay diversas } \\
\text { historias de terror sobre } \\
\text { hechos sucedidos en el } \\
\text { pasado" }\end{array}$ & $\begin{array}{l}\text { Los participantes describen celebraciones } \\
\text { y festividades culturales tradicionales } \\
\text { que se desarrollan de forma anual, desde } \\
\text { el nacimiento del municipio de El Retén } \\
\text { que representan los aportes y herencias } \\
\text { culturales, ancestrales significativos, tanto } \\
\text { de la zona, como de la región, es evidente } \\
\text { la importancia que le dan a las actividades } \\
\text { religiosas católicas y a las demostraciones } \\
\text { artísticas y naturales la comunidad. Como } \\
\text { lo expone (Arévalo, 2004) Tradición, } \\
\text { Patrimonio e Identidad son conceptos } \\
\text { complejos, ambiguos y polisémicos; } \\
\text { porque son construcciones sociales cuyos } \\
\text { significados cambian dependiendo de la } \\
\text { época, el tiempo histórico y según quienes } \\
\text { los empleen y para qué fines los utilicen. }\end{array}$ \\
\hline
\end{tabular}


Entorno Turístico

¿Según se percepción, ¿cuáles son los lugares representativos del Municipio? "la tarima municipal, el parque central y la iglesia", "la reserva natural el Chuval, el puente -la hamaca-, y el bongo" "las canchas de futbol, la alcaldía municipal y las instituciones educativas", "la cancha de la INA y el 26 de julio" "las primeras casas de Él Reten y las reserva natural", "la sede de la casa de la cultura", "la registraduría y el rio" "el parque Simón Bolívar o central y las escuelas de danzas folclóricas”.
En este apartado los estudiantes y docentes de la IED Roque de los Ríos Valle muestran los componentes predominantes del patrimonio de El Retén Magdalena con los lugares municipales representativos de congregación comunitaria, que han perdurado y se han desarrollado a lo largo de la historia y en donde han tenido lugar importantes acontecimientos de sus pobladores.

Evidenciando lo expresado por (Esmoris, 2001) en cuanto a que el patrimonio comprende la herencia compartida entre personas, de bienes materiales (inmuebles o muebles) e inmateriales que son seleccionados por las dimensiones, del sentido de identidad, lo estéticos / artísticos y etnográficos.

El patrimonio cultural en un sentido amplio se puede establecer por: "relatos históricos sobre acontecimientos diversos, bienes arqueológicos, arquitectónicos, archivos y acervos, obras de arte, mobiliario, expresiones artísticas, máquinas, artefactos y objetos singulares y/o representativos de actividades, épocas y estilos." (Esmoris, 2001).

Los participantes manifiestan la importancia de que el municipio cuente con dos ríos cercanos, predominan las concepciones sobre la utilidad en las cosechas y la conservación ambiental, la aplicabilidad en la vida comunitaria y a la calidad del agua de esta zona.

Frente a esto es fundamental exponer la iniciativa de la denominada "el caso de la ruta del sur" en la cual según lo establece (Burbano, 2011) "las decisiones se tomaron teniendo en cuenta a todos los actores. Los estudios realizados siempre contaron con parte práctica y aplicable, se realizó un plan de vida comunitario, acordando el sueño de territorio, no solo del producto turístico, sino de la comunidad a unos años. Los corregimientos están en el rio cauca y la calidad de agua es buena en esta zona. La comunidad es ambientalmente muy consciente y responsable. La zona tiene una cobertura vegetal, productiva y ayuda a conservar la rivera del rio. Y la comunidad tiene policultivo, para consumo propio, pero también para comercializar. Se ofrece a los turistas: Balsaje en el río, avistamiento de aves, cabalgatas, caminatas, gastronomía, entre otras." 


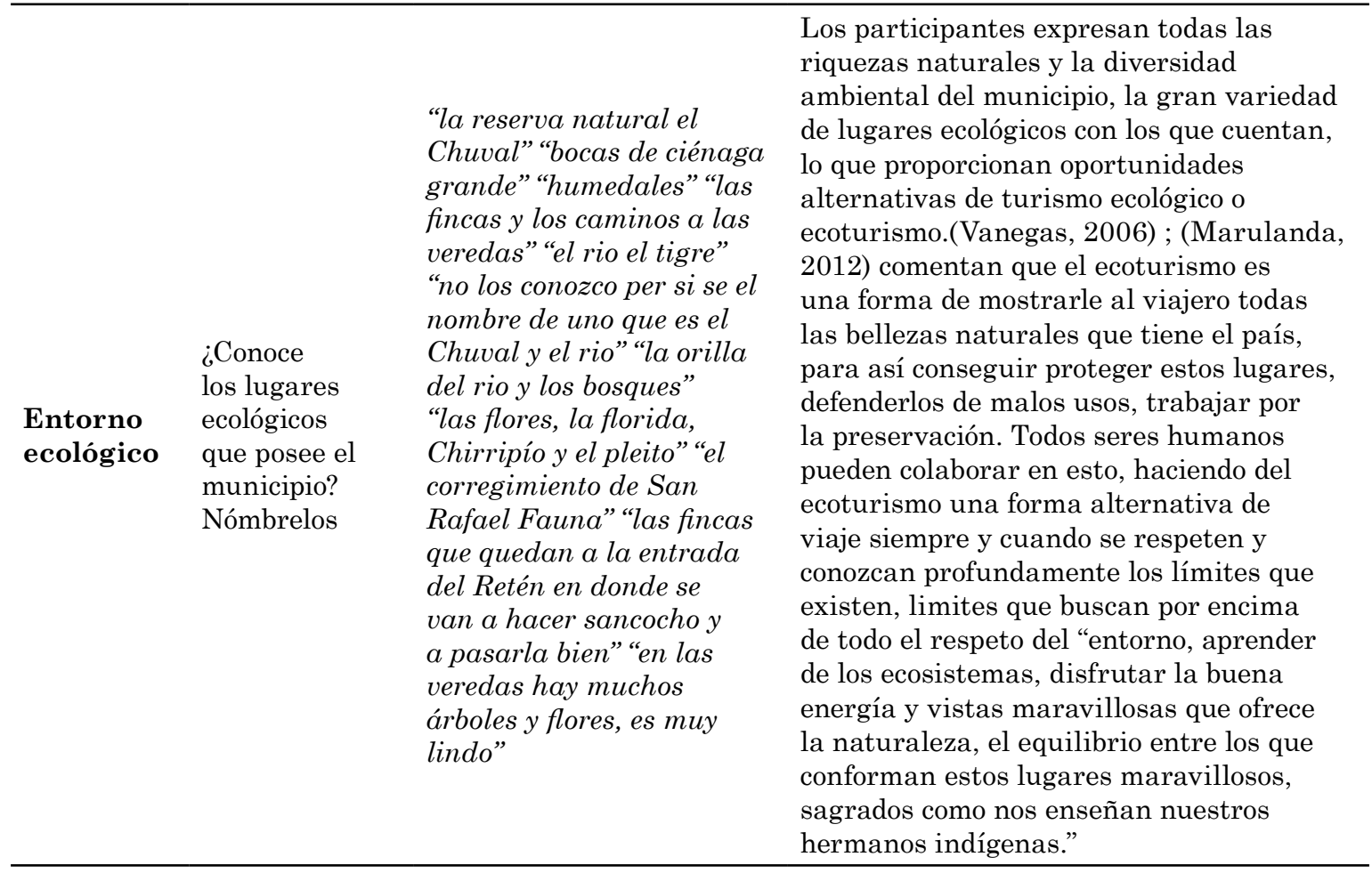

\section{Discusión}

El municipio del Reten Magdalena cuenta con hermosos paisajes, lugares y espacios verdes aptos para la recreación, sin embargo, no cuenta con rutas y guías turísticas, siendo que el turismo trae consigo muchos beneficios, porque, además de generar ingresos, favorece ambientes para la integración familiar, aprovechamiento de recursos y tiempo libre, desarrollo del sentido de pertenencia, etc.

La conclusión es que los docentes deben indagar sobre todos los sitios de interés con que cuenta el municipio, apropiarse de esta información y compartirla con los estudiantes para motivarlos a expandir su horizonte fijándose metas de desarrollo personal y comunitario, porque si bien el municipio cuenta con amplios y lugares aptos para visitar y de los cuales aprende, son los habitantes los que desconoces de las propiedades ventajosas del paisaje, de la cultura y de los recursos ecológicos que serán de gran utilidad no solo para los que allí habitan, sino que también los visitantes puedan sentirse orientados y conozca de lugares como e estudiado.

\section{Referencias}

Arévalo, J. M. (2004). La tradición, el patrimonio y la identidad. España. Obtenido de http://sgpwe.izt. uam.mx/files/users/uami/mcheca/GEOPATRIMONIO/LECTURA2E.pdf 
Avendaño ,I;Cortés O , Guerrero Hilda( 2015) Competencias sociales y tecnologías de la información y la comunicación como factores asociados al desempeño en estudiantes de básica primaria con experiencia de desplazamiento forzado Diversitas: Perspectivas en Psicología, vol. 11, núm. 1, 2015, pp. 13-36 Universidad Santo Tomás Bogotá, Colombia.http://www.scielo.org.co/ pdf/dpp/v11n1/v11n1a02.pdf.

Burbano, A. (19 de febrero de 2011). Comunidad Hostertur. Obtenido de http://comunidad.hosteltur. com/post/2011-02-09-turismo-comunitario-en-colombia-el-caso-dela-ruta-del-sur.html

Burgos, R. (2016). El turismo comunitario como iniciativa de desarrollo local. Caso localidades de Ciudad Bolívar y Usme zona rural de Bogotá. Hallazgos, 13(26). Obtenido de http://revistas.usta.edu.co/index. php/hallazgos/article/view/3162

Campodónico, R., \& Chalar, L. (Julio de 2010). TURISMO: UNA CIENCIA EN CONSTRUCCION. Universidad de Caxias do Sul, 1-15. Obtenido de https://www.ucs.br/ ucs/eventos/seminarios_semintur/ semin_tur_6/arquivos/09/Turismo\%20una $\% 20$ ciencia $\% 20 \mathrm{em} \% 20$ construccion.pdf

Capanegra, C. (2008). Turismo y desarrollo: crecimiento y pobreza. (S. M. Arnaiz Burne, \& A. C. Dachary, Edits.) Buenos Aires, Mar del Plata, Argentina. Obtenido de http:// www.cuc.udg.mx/sites/default/files/ publicaciones/2008\%20-\%20Turismo\%20y\%20Desarrollo.\%20Crecimiento\%20y\%20Pobreza\%20-\%20 interiores.pdf
Esmoris, M. (2001). El patrimonio y las tradiciones culturales. España. Obtenido de http://www.montevideo.gub.uy/sites/default/files/patrimonio.pdf

Guzmán, M. C. (2017). Marco teórico para la construcción de una propuesta de turismo rural comunitario. (U. N. Distancia, Ed.) Revista de investigación agraria y ambiental, 8(1). Obtenido de http://hemeroteca. unad.edu.co/index.php/riaa/article/ view/1841/2060.

Guerrero, H. y Cepeda, M. (2016). El impacto del líder comunitario en el siglo XXI. Barranquilla: Editorial Universitaria de la Costa.

Jiménez, I. V. (2012). La entrevista en la investigación cualitativa: Nuevas tendencias y retos. $R e$ vista Calidad en la Educación Superior, 119-139. Obtenido de http://biblioteca.icap.ac.cr/BLIVI/ COLECCION_UNPAN/BOL_DICIEMBRE_2013_69/UNED/2012/ investigacion_cualitativa.pdf

Reyes, G., Marín, F. y Gutiérrez, C. (2006). Satisfacción del cliente de las posadas turísticas en la Península de Paraguaná, estado Falcón, Venezuela. Multiciencias, 6(3), 271277.

Marulanda, D. (2012). Ecoturismo en Colombia. (D. M. López, Editor) Obtenido de blogspot: http://gestionandari.blogspot.com.co/2012/

Murillo, J., \& Martínez-Garrido, C. (2010). Investigación Etnográfica. (U. A. Madrid, Ed.) Madrid, España. Obtenido de https://www.uam. es/personal_pdi/stmaria/jmurillo/ InvestigacionEE/Presentaciones/ Curso_10/I_Etnografica_Trabajo. pdf 
Natura, F. (09 de Febreo de 2011). Turismo Comunitario en Colombia, el caso de La Ruta del Sur. Obtenido de http:// www.forumnatura.org/2011/02/09/ turismo-comunitario-en-colombiael-caso-de-la-ruta-del-sur/
Vanegas, G. M. (2006). Ecoturismo, instrumento de desarrollo sostenible. Medellín, Colombia. Obtenido de http:// bibliotecadigital.udea.edu.co/bitstream/10495/149/1/EcoturismoInstrumentoDesarrolloSostenible.pdf 